\title{
First detection and genotyping of Enterocytozoon bieneusi in pet fancy rats (Rattus norvegicus) and guinea pigs (Cavia porcellus) in China
}

\author{
Jingsong Wanga, Chaochao Lvª, Diandian Zhao, Runan Zhu, Chen Li, and Weifeng Qian* \\ College of Animal Science and Technology, Henan University of Science and Technology, Luoyang 471003, China
}

Received 29 January 2020, Accepted 21 March 2020, Published online 6 April 2020

\begin{abstract}
Enterocytozoon bieneusi, an obligate intracellular microsporidian parasite, can infect humans and a wide variety of animals worldwide. However, information on the prevalence and molecular characterization of E. bieneusi in pet rats and guinea pigs is lacking. In this study, 325 fecal samples were collected from 152 pet fancy rats and 173 pet guinea pigs purchased from pet shops in Henan and Shandong provinces. The prevalence of E. bieneusi was $11.2 \%$ $(17 / 152)$ in pet fancy rats and 20.2\% (35/173) in pet guinea pigs. Genotypes D $(n=12)$, Peru11 $(n=3)$, S7 $(n=1)$ and SCC-2 $(n=1)$ were identified in pet fancy rats, and genotype S7 $(n=30)$ and a novel genotype PGP $(n=5)$ were identified in pet guinea pigs. The ITS sequence and its phylogenetic analysis showed that the novel genotype PGP was distinctly different; it exhibited less than 50\% similarity to the reference sequences, and did not cluster with any of the known E. bieneusi genotype groups, forming a unique branch between groups 6 and 7. These data suggest that this is a new E. bieneusi genotype group. This is the first report of E. bieneusi infection in pet fancy rats and pet guinea pigs worldwide. The identification of zoonotic genotypes D, Peru11, and S7 suggests that pet fancy rats and guinea pigs can be potential sources of human microsporidiosis.
\end{abstract}

Key words: Enterocytozoon bieneusi, Pet rats, Pet guinea pigs, Genotype, Zoonotic, China.

Résumé - Première détection et génotypage d'Enterocytozoon bieneusi chez des rats (Rattus norvegicus) et des cobayes (Cavia porcellus) de compagnie en Chine. Enterocytozoon bieneusi, un parasite microsporidien intracellulaire obligatoire, peut infecter les humains et une grande variété d'animaux dans le monde. Cependant, les informations sur la prévalence et la caractérisation moléculaire d'E. bieneusi chez les rats et les cobayes de compagnie manquaient. Dans cette étude, 325 échantillons de matières fécales ont été prélevés de 152 rats et 173 cobayes achetés dans des animaleries dans les provinces du Henan et du Shandong. La prévalence d'E. bieneusi était de $11,2 \%(17 / 152)$ chez les rats et de 20,2 \% (35/173) chez les cobayes. Les génotypes D $(n=12)$, Peru11 $(n=3)$, S7 $(n=1)$ et SCC-2 $(n=1)$ ont été identifiés chez des rats de compagnie, et le génotype S7 $(n=30)$ et un nouveau génotype PGP $(n=5)$ ont été identifiés chez des cobayes de compagnie. La séquence d'ITS et son analyse phylogénétique ont montré que le nouveau génotype PGP était nettement différent ; la séquence présentait moins de $50 \%$ de similitude avec les séquences de référence et ne se regroupait avec aucun des groupes de génotypes connus d'E. bieneusi, formant une branche unique entre les groupes 6 et 7 ; ces données suggèrent qu'il s'agit d'un nouveau groupe de génotype d'E. bieneusi. Ceci est le premier signalement d'infection par E. bieneusi chez des rats et des cobayes de compagnie dans le monde. L'identification des génotypes zoonotiques $\mathrm{D}$, Peru11 et $\mathrm{S} 7$ suggère que les rats et les cobayes de compagnie peuvent être des sources potentielles de microsporidiose humaine.

\section{Introduction}

Enterocytozoon bieneusi, a unicellular and obligate intracellular pathogen, has an extensive host range and has been identified in humans, livestock, companion animals, and wildlife, as well as in wastewater [17, 23]. Enterocytozoon bieneusi

\footnotetext{
*Corresponding author: qwf2012@yeah. net
}

${ }^{\mathrm{a}}$ These authors contributed equally to this work. infection can cause self-limiting diarrhea, malabsorption, and wasting in immunocompetent hosts and life-threatening diarrhea in immunocompromized individuals [10]. Humans and animals can acquire infection via fecal-oral transmission of spores from infected individuals through direct contact or by consumption of contaminated food or water [17].

Genotyping based on the internal transcribed spacer (ITS) region of the rRNA gene has identified 11 major phylogenetic groups and more than 470 genotypes of $E$. bieneusi from 
Table 1. Prevalence and genotypes of Enterocytozoon bieneusi in pet fancy rats (Rattus norvegicus) in Henan and Shandong provinces, China.

\begin{tabular}{lccl}
\hline Characteristics & No. of animals & No. positive (\%) & Genotypes (no.) \\
\hline $\begin{array}{l}\text { Region } \\
\text { Luoyang, Henan }\end{array}$ & & & \\
$\quad$ Pet shop 1 & 38 & $4(10.5)$ & D (2), Peru11(2) \\
$\quad$ Pet shop 2 & 30 & $2(6.7)$ & D (1), S7 (1) \\
$\quad$ Subtotal & 68 & $6(8.8)$ & D (3), Peru11(2), S7 (1) \\
Weifang, Shandong & & & \\
$\quad$ Pet shop 4 & 44 & $7(15.9)$ & D (6), SCC-2(1) \\
$\quad$ Pet shop 5 & 40 & $4(10.0)$ & D (3), Peru11(1) \\
$\quad$ Subtotal & 84 & $11(13.1)$ & D (9), Peru11(1), SCC-2 (1) \\
Total & 152 & $17(11.2)$ & D (12), Peru11(3), S7 (1), SCC-2 (1) \\
Age (weeks) & & & \\
$\quad 4-6$ & 105 & $13(12.4)$ & D (9), Peru11(2), S7 (1), SCC-2 (1) \\
$\quad$ 7-10 & 47 & $4(8.5)$ & D (3), Peru11(1) \\
Sex & & & \\
$\quad$ Male & 85 & $11(12.9)$ & D (8), Peru11(2), S7 (1) \\
Female & 67 & $6(9.0)$ & D (4), Peru11(1), SCC-2 (1) \\
\hline
\end{tabular}

various hosts [17]. To date, more than 60 E. bieneusi genotypes have been identified in rodents worldwide $[6,8,12,17,24,26$, 30, 34]. For rats (Rattus spp.), only three surveys have focused on the molecular characterization of E. bieneusi in wild rats in Iran and China, and six genotypes (D, M, Peru6, CD6, BEB6, and CHG2) have been identified [24, 30, 34]. Only one published article has reported genotype peru16 from household guinea pigs in Peru [3].

Fancy rats, Rattus norvegicus forma domestica, are rodents belonging to the order Rodentia and family Muridae. Fancy rats have been bred as pets at least since the late 19th century; they are considered to be intelligent, playful, and trainable animals (http://www.afrma.org/). In recent years, fancy rats have become a very popular pet in China. Pet rodents can be hosts to several zoonotic pathogens, including viruses, bacteria, and parasites [20]; zoonotic transmission of E. bieneusi to a child from household guinea pigs has been reported [3]. However, no literature is available about the prevalence and genetic characteristics of E. bieneusi in pet rats and pet guinea pigs. Therefore, the aim of the present study was to determine the prevalence and genotypes of E. bieneusi in these animals and to assess its zoonotic potential.

\section{Materials and methods \\ Ethics statement}

The research protocol was reviewed and approved by the Research Ethics Committee of Henan University of Science and Technology.

\section{Sample collection}

Between September 2018 and October 2019, 152 pet fancy rats and 173 pet guinea pigs were purchased from six pet shops in Luoyang, Henan and Weifang, Shandong, China (Tables 1 and 2). Upon arrival in the laboratory, each animal was immediately placed into a single clean plastic box for collection of fresh feces. A single sample was collected from each animal.
Table 2. Prevalence and genotypes of Enterocytozoon bieneusi in pet guinea pigs (Cavia porcellus) in Henan and Shandong provinces, China.

\begin{tabular}{lccl}
\hline Characteristics & $\begin{array}{c}\text { No. of } \\
\text { animals }\end{array}$ & $\begin{array}{c}\text { No. positive } \\
(\%)\end{array}$ & \multicolumn{1}{c}{$\begin{array}{c}\text { Genotypes } \\
\text { (no.) }\end{array}$} \\
\hline $\begin{array}{l}\text { Region } \\
\text { Luoyang, Henan }\end{array}$ & & & \\
$\quad$ Pet shop 1 & 35 & $5(14.3)$ & S7 (5) \\
$\quad$ Pet shop 2 & 32 & $7(21.9)$ & S7 (5), PGP (2) \\
$\quad \begin{array}{l}\text { Pet shop 3 } \\
\text { Subtotal }\end{array}$ & 35 & $4(11.4)$ & S7 (4) \\
Weifang, Shandong & 102 & $16(15.7)$ & S7 (14), PGP (2) \\
$\quad$ Pet shop 5 & 35 & $6(17.1)$ & S7 (6) \\
$\quad \begin{array}{l}\text { Pet shop 6 } \\
\text { Subtotal }\end{array}$ & 36 & $13(36.1)$ & S7 (10), PGP (3) \\
Total & 71 & $19(26.8)$ & S7 (16), PGP (3) \\
Age (months) & 173 & $35(20.2)$ & S7 (30), PGP (5) \\
$\quad$ 1-3 & 122 & $29(23.8)$ & S7 (26), PGP (3) \\
$\quad$ 4-8 & 51 & $6(11.8)$ & S7 (4), PGP (2) \\
Sex & & & \\
$\quad$ Male & 76 & $16(21.1)$ & S7 (14), PGP (2) \\
$\quad$ Female & 97 & $19(19.6)$ & S7 (16), PGP (3) \\
\hline
\end{tabular}

All the specimens were refrigerated at $4{ }^{\circ} \mathrm{C}$ and DNA was extracted within one week. Only young pet fancy rats (4-10 week-old) and 1-8-month-old pet guinea pigs were available in these pet shops. All pet fancy rats and guinea pigs examined in this study were asymptomatic at the time of sample collection, and information on region, age, and sex of these animals was recorded.

\section{DNA extraction}

Each specimen was washed with distilled water by centrifugation for $10 \mathrm{~min}$ at $3000 \times g$ at room temperature. Before DNA extraction, $200 \mathrm{mg}$ of each fecal sample was added to a $2 \mathrm{~mL}$ microcentrifuge tube containing $200 \mathrm{mg}$ of glass beads, and were vortexed at maximum speed until the fecal samples were completely homogenized. Genomic DNA was extracted using an E.Z.N.A. Stool DNA Kit (Omega Bio-tek Inc., 
Table 3. Prevalence and genotypes of Enterocytozoon bieneusi in rats (Rattus spp.) and guinea pigs worldwide.

\begin{tabular}{|c|c|c|c|c|c|c|}
\hline Country & Host & $\begin{array}{c}\text { No. } \\
\text { examined }\end{array}$ & $\begin{array}{c}\text { No. } \\
\text { positive }\end{array}$ & $\begin{array}{c}\text { Prevalence } \\
(\%)\end{array}$ & Genotypes (no.) & Reference \\
\hline \multirow[t]{2}{*}{ Iran } & Wild rats ( $R$. norvegicus) & 146 & 13 & 8.9 & $\mathrm{D}^{*}(11), \mathrm{M}^{*}(2)$ & [24] \\
\hline & Wild rats $(R$. rattus $)$ & 14 & 1 & 7.1 & $\mathrm{D}(1)$ & \\
\hline China & Wild rats ( $R$. norvegicus) & 242 & 19 & 7.9 & D (17), Peru6* (2) & [34] \\
\hline China & Wild rats ( $R$. norvegicus) & 199 & 8 & 4.0 & CD6 (3) $)^{\mathrm{a}}, \mathrm{BEB}^{*}$ (2), D (2), CHG2 (1) & [30] \\
\hline China & Pet rats ( $R$. norvegicus) & 152 & 17 & 11.2 & D (12), Peru11* (3), S7*(1), SCC-2 (1) & This study \\
\hline Peru & Household guinea pigs & 67 & 10 & 14.9 & Peru16* (10) & [3] \\
\hline China & Pet guinea pigs (Cavia porcellus) & 173 & 35 & 20.2 & S7 (30), novel genotype PGP (5) & This study \\
\hline
\end{tabular}

a Genotype CD6 is a synonym of genotype CHG14, and genotype S7 is a synonym of genotype CHY1, based on the nomenclature system established by Santin and Fayer [22].

Known zoonotic genotypes: D, M, Peru6, BEB6, Peru11, Peru16, and S7.

Norcross, GA, USA), according to the manufacturer's instructions. The extracted DNA was kept at $-20{ }^{\circ} \mathrm{C}$ before being used in PCR analysis.

\section{PCR amplification}

Enterocytozoon bieneusi was examined by nested PCR targeting a $\sim 390-\mathrm{bp}$ fragment of the ITS region, as previously described [2]. The primers were EBITS3 (5'GGTCATAGGGATGAAGAG-3 $3^{\prime}$ ) and EBITS4 (5'-TTCGAGTTCTTTCGCGCTC- $\left.3^{\prime}\right)$ as external primers and EBITS1 $\left(5^{\prime}-\right.$ GCTCTGAATATCTATGGCT-3') and EBITS2.4 (5'ATCGCCGACGGATCCAAGTG- $3^{\prime}$ ) as internal primers. TransStart ${ }^{\circledR}$ Taq DNA Polymerase (TransGen Biotech, Beijing, China) was used for PCR amplifications. The cycling conditions for PCRs were: $94{ }^{\circ} \mathrm{C}$ for $5 \mathrm{~min}$; followed by 35 cycles of $94{ }^{\circ} \mathrm{C}$ for $30 \mathrm{~s}, 57{ }^{\circ} \mathrm{C}$ (primary PCR) or $55^{\circ} \mathrm{C}$ (secondary PCR) for $30 \mathrm{~s}$, and $72{ }^{\circ} \mathrm{C}$ for $40 \mathrm{~s}$; followed by $72{ }^{\circ} \mathrm{C}$ for $7 \mathrm{~min}$. Positive and negative controls were included in each PCR analysis.

\section{Sequencing and phylogenetic analysis}

Two-directional sequencing of positive PCR products was done by Sangon Biotech Co. Ltd., (Shanghai, China). The obtained nucleotide sequences were aligned with available sequences in GenBank, using ClustalX 2.1 (http://www. clustal.org/) [15]. Genotypes of E. bieneusi were determined based on $\sim 243$ bp of the ITS region, according to the established nomenclature system [22]. A neighbor-joining tree was generated using MEGA7 software (http://www.megasoftware. net/) [14]. The evolutionary distances were computed using the maximum composite likelihood method, and the reliability of branches in the tree was assessed by bootstrap analysis using 1000 replicates.

\section{Statistical analysis}

Chi-square analysis was performed to assess the correlation between the prevalence of $E$. bieneusi and the age, sex, and region of pet fancy rats and guinea pigs using SPSS, version 17.0 (Statistical Package for the Social Sciences).

\section{Nucleotide sequence accession numbers}

Unique ITS nucleotide sequences of $E$. bieneusi obtained from pet fancy rats and guinea pigs in this study were deposited in the GenBank database under accession numbers MN550998-MN551001 and MN998614-MN998615, respectively.

\section{Results and discussion}

In the present study, E. bieneusi was detected by PCR in 17 (11.2\%) of 152 pet fancy rats and $35(20.2 \%)$ of 173 pet guinea pigs. To our knowledge, this is the first report of E. bieneusi infection in pet rats and pet guinea pigs worldwide. To date, there have been three studies focusing on E. bieneusi infection in wild rats in Iran and China [24, 30, 34] (Table 3). In this study, the prevalence of $E$. bieneusi in pet fancy rats was slightly higher than that $(4.0 \%-8.9 \%)$ in wild rats in the above-mentioned reports. The prevalence of E. bieneusi in pet guinea pigs in this study was higher than that $(14.9 \%$, 10/67) in household guinea pigs in Peru [3], and also higher than other pet rodents, such as pet chinchillas (3.6\%), pet squirrels $(16.7 \%)$ and chipmunks $(17.6 \%)$ [7, 8, 21].

In both pet fancy rats and guinea pigs, although the prevalences of $E$. bieneusi in younger animals and those from Weifang, Shandong were higher than those in older animals and animals from Luoyang, Henan (Tables 1 and 2), the differences in prevalence in both species between different regions, ages and sex groups were not significant $(p>0.05)$. This finding was consistent with the observations reported in a previous study on pet red-bellied tree squirrels in China [7].

In the 17 E. bieneusi ITS-positive samples from pet fancy rats in this study, four known genotypes were identified; genotype $\mathrm{D}(n=12)$ was the dominant genotype, followed by Peru11 $(n=3), \mathrm{S} 7(n=1)$, and SCC-2 $(n=1)$ (Table 1). For pet guinea pigs, two genotypes were identified, including the predominant genotype S7 $(n=30,85.7 \%)$ and a novel genotype (named PGP, $n=5$ ) (Table 2). Until now, molecular studies of E. bieneusi in rats have been limited to three reports in wild rats ( $R$. norvegicus and $R$. rattus) in Iran and China, and a total of six genotypes were identified, including genotypes D, CD6 (synonyms: CHG14), Peru6, M, BEB6, and CHG2 [24, 30, 34] (Table 3). For guinea pigs, only one survey 


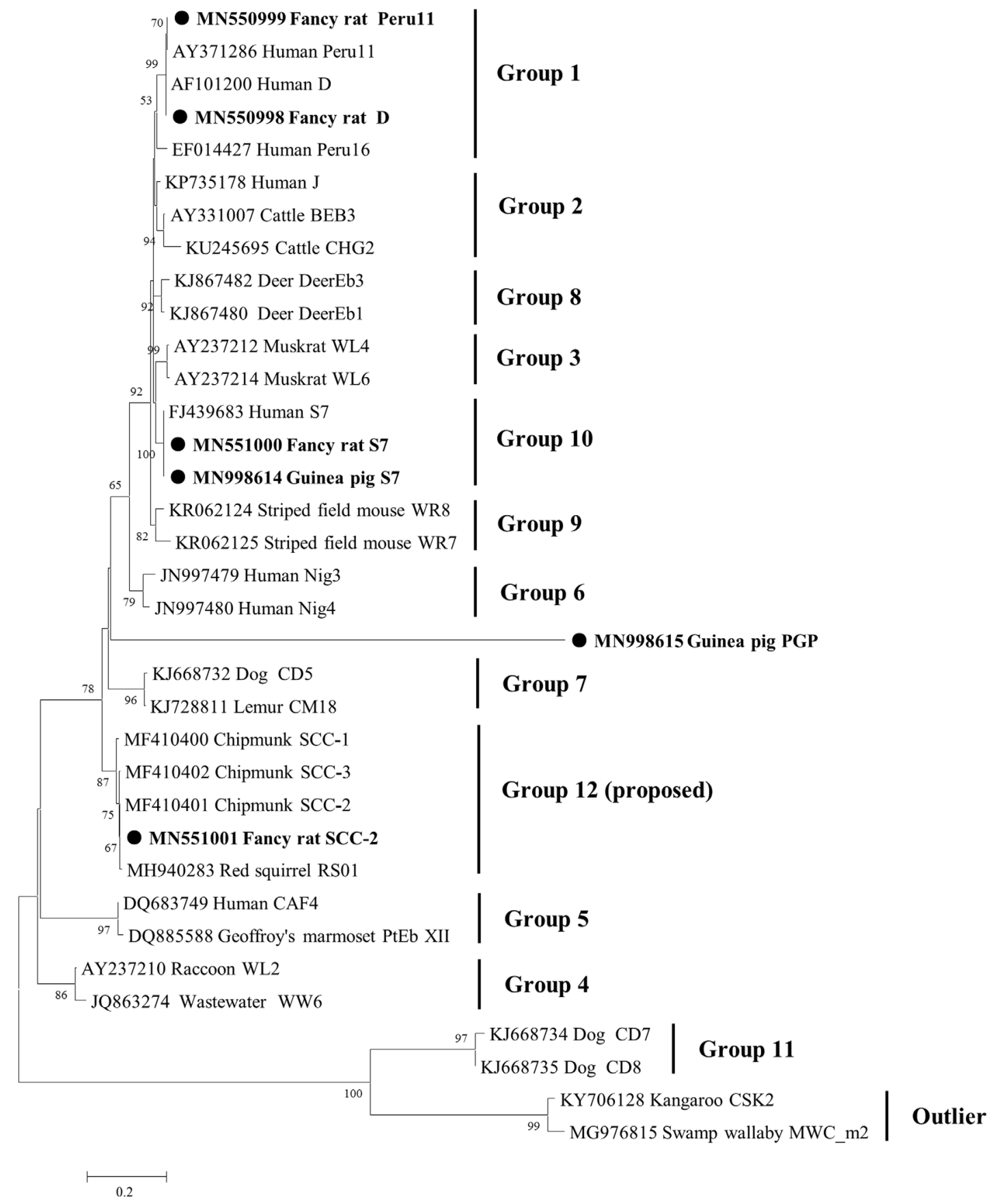

Figure 1. Phylogenetic relationships among the genotypes of E. bieneusi identified in this study and other known genotypes, as inferred by a neighbor-joining analysis of the ITS region. Bootstrap values greater than $50 \%$ from 1000 pseudoreplicates are shown. The genotypes identified in this study are indicated by closed circles.

conducted in Peru identified genotype peru16 from household guinea pigs (Table 3) [3], and this genotype was not detected in the present study.

In this study, genotype D was the most prevalent genotype in pet fancy rats, which is consistent with two previous reports $(85.7 \%$ and $89.5 \%)$ from wild rats [24, 34], as well as other rodents such as pet red-bellied tree squirrels (Callosciurus erythraeus) (75.0\%), pet red squirrels (Sciurus vulgaris) (44.3\%), and domestic bamboo rats (Rhizomys sinensis) $(77.3 \%)[6,7,26]$. Genotype D is considered an important zoonotic genotype worldwide [17]. In China, genotype D has been identified in immunocompromized patients and in children with diarrhea [18, 27-29, 32]. Genotype D has also been identified in a wide range of animal hosts in China, including non-human primates, rodents (mice, rats, squirrels, chipmunks, chinchillas, and bamboo rats), other mammals (pigs, cattle, sheep, goats, alpacas, horses, donkeys, rabbits, dogs, cats, foxes, deer, takins, minks, raccoon dogs, raccoons, lions, and hippos), and birds, as well as in water samples [6, 8, 9, 11, 17, 24, 26, 30, 31, 33, 34].

In the present study, genotype Peru11, a zoonotic genotype, was identified in pet fancy rats for the first time. This genotype 
has been found previously in humans in Peru, China and Thailand, non-human primates in Kenya and China, raccoons, voles and cottontails in the United States, chickens in Brazil, cats in Spain, and minks and water in China [4, 5, 17, 33]. Genotype SCC-2 was reported previously in pet chipmunks and squirrels in China $[6,8]$, and was found in pet fancy rats for the first time. Genotype S7 (synonyms: CHY1) was originally reported in a patient in the Netherlands [25], and recently identified in a yak and pet chipmunks in China [8, 16]. This genotype was identified in a fancy rat; moreover, it was predominant in pet guinea pigs in this study, suggesting that guinea pig might be an important reservoir host of genotype S7. More studies are needed to understand the host range and public health importance of genotypes S7 and SCC-2.

In the phylogenetic tree of the E. bieneusi ITS region (Fig. 1), genotypes D and Peru11 were clustered into group 1 with strong zoonotic potential [17], and genotype S7 was clustered into group 10. Genotype SCC-2 belonged to a group which includes several chipmunk and squirrel-derived genotypes such as SCC-1-3 and RS01. Sequence and phylogenetic analysis showed that the novel genotype PGP was distinctly different. Genotype PGP exhibited less than 50\% sequence similarity to the reference sequences from the known E. bieneusi genotype groups and outliers, i.e., $45.5 \%$ similarity to genotype CM18 in group 7, and less than $30 \%$ as compared with those in group 11 and outliers. The novel genotype PGP identified in pet guinea pigs did not cluster with any of the known E. bieneusi genotype groups, and formed a unique branch which was located at an intermediate position between groups 6 and 7 (Fig. 1). These data suggest that the genetic variability of E. bieneusi is broad, and indicate the presence of a new E. bieneusi genotype group; similar observations have been reported in previous studies $[1,13,19]$. Further studies on more samples collected from different regions should be conducted to understand the genetic diversity of E. bieneusi from rodents in China.

\section{Conclusions}

This is the first report of E. bieneusi infection in pet fancy rats and pet guinea pigs. Five genotypes (D, Peru11, S7, SCC2 , and a novel genotype PGP) were identified in this study, and genotypes D and S7 were the dominant genotypes in pet fancy rats and guinea pigs, respectively. Rats (Rattus norvegicus) are a new host of E. bieneusi genotypes Peru11, S7, and SCC-2, and guinea pigs might be an important reservoir host of genotype S7. The identification of three zoonotic genotypes (D, Peru11, and S7) suggests that pet fancy rats and guinea pigs may be the sources of E. bieneusi infection in humans. Therefore, pet owners, especially children, should be educated to take precautions to reduce the transmission risk.

\section{Competing interests}

The authors declare that they have no competing interests.

Acknowledgements. This study was supported by the National Natural Science Foundation of China (Grant No. 31502053), Student Research Training Program (SRTP) in Henan University of Science and Technology (2019392) and Henan Province (S201910464052), and Key Scientific Research Projects of Higher Education Institutions in Henan Province (Grant No. 20A230001).

\section{References}

1. Breton J, Bart-Delabesse E, Biligui S, Carbone A, Seiller X, Okome-Nkoumou M, Nzamba C, Kombila M, Accoceberry I, Thellier M. 2007. New highly divergent rRNA sequence among biodiverse genotypes of Enterocytozoon bieneusi strains isolated from humans in Gabon and Cameroon. Journal of Clinical Microbiology, 45, 2580-2589.

2. Buckholt MA, Lee JH, Tzipori S. 2002. Prevalence of Enterocytozoon bieneusi in swine: an 18-month survey at a slaughterhouse in Massachusetts. Applied and Environmental Microbiology, 68, 2595-2599.

3. Cama VA, Pearson J, Cabrera L, Pacheco L, Gilman R, Meyer S, Ortega Y, Xiao L. 2007. Transmission of Enterocytozoon bieneusi between a child and guinea pigs. Journal of Clinical Microbiology, 45, 2708-2710.

4. Cong W, Qin SY, Meng QF. 2018. Molecular characterization and new genotypes of Enterocytozoon bieneusi in minks (Neovison vison) in China. Parasite, 25, 34.

5. Dashti A, Santin M, Cano L, de Lucio A, Bailo B, de Mingo MH, Koster PC, Fernandez-Basterra JA, Aramburu-Aguirre J, LopezMolina N, Fernandez-Crespo JC, Calero-Bernal R, Carmena D. 2019. Occurrence and genetic diversity of Enterocytozoon bieneusi (Microsporidia) in owned and sheltered dogs and cats in Northern Spain. Parasitology Research, 118, 2979-2987.

6. Deng L, Chai Y, Luo R, Yang L, Yao J, Zhong Z, Wang W, Xiang L, Fu H, Liu H, Zhou Z, Yue C, Chen W, Peng G. 2020. Occurrence and genetic characteristics of Cryptosporidium spp. and Enterocytozoon bieneusi in pet red squirrels (Sciurus vulgaris) in China. Scientific Reports, 10, 1026.

7. Deng L, Li W, Yu X, Gong C, Liu X, Zhong Z, Xie N, Lei S, Yu J, Fu H, Chen H, Xu H, Hu Y, Peng G. 2016. First report of the human-pathogenic Enterocytozoon bieneusi from red-bellied tree squirrels (Callosciurus erythraeus) in Sichuan, China. PLoS One, 11, e0163605.

8. Deng L, Li W, Zhong Z, Chai Y, Yang L, Zheng H, Wang W, Fu H, He M, Huang X, Zuo Z, Wang Y, Cao S, Liu H, Ma X, Wu K, Peng G. 2018. Molecular characterization and new genotypes of Enterocytozoon bieneusi in pet chipmunks (Eutamias asiaticus) in Sichuan province, China. BMC Microbiology, 18, 37.

9. Deng L, Yue C, Chai Y, Wang W, Su X, Zhou Z, Wang L, Li L, Liu H, Zhong Z, Cao S, Hu Y, Fu H, Peng G. 2019. New genotypes and molecular characterization of Enterocytozoon bieneusi in pet birds in Southwestern China. International Journal for Parasitology: Parasites and Wildlife, 10, 164-169.

10. Didier ES, Weiss LM. 2006. Microsporidiosis: current status. Current Opinion in Infectious Diseases, 19, 485-492.

11. Feng SY, Chang H, Luo J, Huang JJ, He HX. 2019. First report of Enterocytozoon bieneusi and Cryptosporidium spp. in peafowl (Pavo cristatus) in China. International Journal for Parasitology: Parasites and Wildlife, 9, 1-6.

12. Gui BZ, Zou Y, Chen YW, Li F, Jin YC, Liu MT, Yi JN, Zheng WB, Liu GH. 2020. Novel genotypes and multilocus genotypes of Enterocytozoon bieneusi in two wild rat species in China: potential for zoonotic transmission. Parasitology Research, 119, 283-290.

13. Karim MR, Wang R, Dong H, Zhang L, Li J, Zhang S, Rume FI, Qi M, Jian F, Sun M, Yang G, Zou F, Ning C, Xiao L. 2014. Genetic polymorphism and zoonotic potential of Enterocytozoon bieneusi from nonhuman primates in China. Applied and Environmental Microbiology, 80, 1893-1898. 
14. Kumar S, Stecher G, Tamura K. 2016. MEGA7: Molecular Evolutionary Genetics Analysis Version 7.0 for Bigger Datasets. Molecular Biology and Evolution, 33, 1870-1874.

15. Larkin MA, Blackshields G, Brown NP, Chenna R, McGettigan PA, McWilliam H, Valentin F, Wallace IM, Wilm A, Lopez R, Thompson JD, Gibson TJ, Higgins DG. 2007. Clustal W and Clustal X version 2.0. Bioinformatics, 23, 2947-2948.

16. Li J, Qi M, Chang Y, Wang R, Li T, Dong H, Zhang L. 2015. Molecular characterization of Cryptosporidium spp., Giardia duodenalis, and Enterocytozoon bieneusi in captive wildlife at Zhengzhou zoo, China. Journal of Eukaryotic Microbiology, 62, 833-839.

17. Li W, Feng Y, Santin M. 2019. Host specificity of Enterocytozoon bieneusi and public health implications. Trends in Parasitology, 35, 436-451.

18. Liu H, Jiang Z, Yuan Z, Yin J, Wang Z, Yu B, Zhou D, Shen Y, Cao J. 2017. Infection by and genotype characteristics of Enterocytozoon bieneusi in HIV/AIDS patients from Guangxi Zhuang autonomous region, China. BMC Infectious Diseases, 17, 684.

19. Mathis A, Breitenmoser AC, Deplazes P. 1999. Detection of new Enterocytozoon genotypes in faecal samples of farm dogs and a cat. Parasite, 6, 189-193.

20. Meerburg BG, Singleton GR, Kijlstra A. 2009. Rodent-borne diseases and their risks for public health. Critical Reviews in Microbiology, 35, 221-270.

21. Qi M, Luo N, Wang H, Yu F, Wang R, Huang J, Zhang L. 2015. Zoonotic Cryptosporidium spp. and Enterocytozoon bieneusi in pet chinchillas (Chinchilla lanigera) in China. Parasitology International, 64, 339-341.

22. Santin M, Fayer R. 2009. Enterocytozoon bieneusi genotype nomenclature based on the internal transcribed spacer sequence: a consensus. Journal of Eukaryotic Microbiology, 56, 34-38.

23. Santin M, Fayer R. 2011. Microsporidiosis: Enterocytozoon bieneusi in domesticated and wild animals. Research in Veterinary Science, 90, 363-371.

24. Tavalla M, Kazemi F, Mardani-Kateki M, Abdizadeh R. 2018. Molecular diagnosis of Enterocytozoon bieneusi and Encephalitozoon spp. in wild rats of southwest of Iran. Jundishapur Journal of Microbiology, 11, e55961.

25. ten Hove RJ, Van Lieshout L, Beadsworth MB, Perez MA, Spee K, Claas EC, Verweij JJ. 2009. Characterization of genotypes of
Enterocytozoon bieneusi in immunosuppressed and immunocompetent patient groups. Journal of Eukaryotic Microbiology, 56, 388-393.

26. Wang H, Liu Q, Jiang X, Zhang Y, Zhao A, Cui Z, Li D, Qi M, Zhang L. 2019. Dominance of zoonotic genotype D of Enterocytozoon bieneusi in bamboo rats (Rhizomys sinensis). Infection, Genetics and Evolution, 73, 113-118.

27. Wang L, Xiao L, Duan L, Ye J, Guo Y, Guo M, Liu L, Feng Y. 2013. Concurrent infections of Giardia duodenalis, Enterocytozoon bieneusi, and Clostridium difficile in children during a cryptosporidiosis outbreak in a pediatric hospital in China. PLoS Neglected Tropical Diseases, 7, e2437.

28. Wang L, Zhang H, Zhao X, Zhang L, Zhang G, Guo M, Liu L, Feng Y, Xiao L. 2013. Zoonotic Cryptosporidium species and Enterocytozoon bieneusi genotypes in HIV-positive patients on antiretroviral therapy. Journal of Clinical Microbiology, 51, $557-563$.

29. Wang T, Fan Y, Koehler AV, Ma G, Li T, Hu M, Gasser RB. 2017. First survey of Cryptosporidium, Giardia and Enterocytozoon in diarrhoeic children from Wuhan, China. Infection, Genetics and Evolution, 51, 127-131.

30. Yu F, Qi M, Zhao Z, Lv C, Wang Y, Wang R, Zhang L. 2019. The potential role of synanthropic rodents and flies in the transmission of Enterocytozoon bieneusi on a dairy cattle farm in China. Journal of Eukaryotic Microbiology, 66, 435-441.

31. Zhang Q, Wang H, Zhao A, Zhao W, Wei Z, Li Z, Qi M. 2019. Molecular detection of Enterocytozoon bieneusi in alpacas (Vicugna pacos) in Xinjiang, China. Parasite, 26, 31.

32. Zhang W, Ren G, Zhao W, Yang Z, Shen Y, Sun Y, Liu A, Cao J. 2017. Genotyping of Enterocytozoon bieneusi and subtyping of Blastocystis in cancer patients: relationship to diarrhea and assessment of zoonotic transmission. Frontiers in Microbiology, 8, 1835.

33. Zhang XX, Jiang RL, Ma JG, Xu C, Zhao Q, Hou G, Liu GH. 2018. Enterocytozoon bieneusi in minks (Neovison vison) in Northern China: A Public Health Concern. Frontiers in Microbiology, 9, 1221.

34. Zhao W, Wang J, Ren G, Yang Z, Yang F, Zhang W, Xu Y, Liu A, Ling H. 2018. Molecular characterizations of Cryptosporidium spp. and Enterocytozoon bieneusi in brown rats (Rattus norvegicus) from Heilongjiang Province, China. Parasites \& Vectors, 11, 313.

Cite this article as: Wang J, Lv C, Zhao D, Zhu R, Li C \& Qian W. 2020. First detection and genotyping of Enterocytozoon bieneusi in pet fancy rats (Rattus norvegicus) and guinea pigs (Cavia porcellus) in China. Parasite 27, 21.

\section{PARASTE}

An international open-access, peer-reviewed, online journal publishing high quality papers on all aspects of human and animal parasitology

Reviews, articles and short notes may be submitted. Fields include, but are not limited to: general, medical and veterinary parasitology; morphology, including ultrastructure; parasite systematics, including entomology, acarology, helminthology and protistology, and molecular analyses; molecular biology and biochemistry; immunology of parasitic diseases; host-parasite relationships; ecology and life history of parasites; epidemiology; therapeutics; new diagnostic tools.

All papers in Parasite are published in English. Manuscripts should have a broad interest and must not have been published or submitted elsewhere. No limit is imposed on the length of manuscripts.

Parasite (open-access) continues Parasite (print and online editions, 1994-2012) and Annales de Parasitologie Humaine et Comparée (1923-1993) and is the official journal of the Société Française de Parasitologie. 